\title{
Detector design for active fault diagnosis in closed-loop systems
}

\author{
Sekunda, André Krabdrup; Niemann, Hans Henrik; Poulsen, Niels Kjølstad
}

Published in:

International Journal of Adaptive Control and Signal Processing

Link to article, DOI:

10.1002/acs.2858

Publication date:

2018

Document Version

Peer reviewed version

Link back to DTU Orbit

Citation (APA):

Sekunda, A. K., Niemann, H. H., \& Poulsen, N. K. (2018). Detector design for active fault diagnosis in closedloop systems. International Journal of Adaptive Control and Signal Processing. https://doi.org/10.1002/acs.2858

\section{General rights}

Copyright and moral rights for the publications made accessible in the public portal are retained by the authors and/or other copyright owners and it is a condition of accessing publications that users recognise and abide by the legal requirements associated with these rights.

- Users may download and print one copy of any publication from the public portal for the purpose of private study or research.

- You may not further distribute the material or use it for any profit-making activity or commercial gain

- You may freely distribute the URL identifying the publication in the public portal

If you believe that this document breaches copyright please contact us providing details, and we will remove access to the work immediately and investigate your claim. 


\title{
Detector design for active fault diagnosis in closed-loop systems
}

\author{
A. K. Sekunda'10 | H. H. Niemann'미 | N. K. Poulsen ${ }^{2}$
}

${ }^{1}$ Department of Electrical Engineering, Automation and Control, Technical University of Denmark, Kongens Lyngby, Denmark

${ }^{2}$ Department of Applied Mathematics and Computer Science, Technical University of Denmark, Kongens Lyngby, Denmark

\section{Correspondence}

AK Sekunda, Department of Electrical Engineering, Automation and Control, Technical University of Denmark, Kongens Lyngby, Denmark.

Email: aksek@elektro.dtu.dk

\begin{abstract}
Summary
Fault diagnosis of closed-loop systems is extremely relevant for high-precision equipment and safety critical systems. Fault diagnosis is usually divided into 2 schemes: active and passive fault diagnosis. Recent studies have highlighted some advantages of active fault diagnosis based on dual Youla-Jabr-Bongiorno-Kucera parameters. In this paper, a method for closed-loop active fault diagnosis based on statistical detectors is given using dual Youla-Jabr-Bongiorno-Kucera parameters. The goal of this paper is 2-fold. First, the authors introduce a method for measuring a residual signal subject to white noise. Second, an optimal detector design is presented for single and multiple faults using the amplitude and phase shift of the residual signal to conduct diagnosis. Here, both the optimal case of a perfect model and the suboptimal case of a model with uncertainties are discussed. The method is successfully tested on a simulated system with parametric faults.
\end{abstract}

\section{KEYWORDS}

active fault diagnosis, closed-loop systems, statistical test, YJBK parameters

\section{1 | INTRODUCTION}

Fault diagnosis has been an intensively studied subject, which has branched into 2 subgroups: passive $e^{1-3}$ and active $e^{4-6}$ fault diagnosis. Broadly speaking, passive fault diagnosis obtains information of faults by the use of residual signals generated by comparing input-output data, ${ }^{7}$ whereas active fault diagnosis uses auxiliary signals to obtain extra information about the state of a system to use for fault diagnosis. So far, most active fault diagnosis solutions have been based on the assumption of an open-loop system description; however, fault diagnosis of closed-loop systems is more relevant for critical systems. For open-loop processes, it is usually possible to assume the characteristics of the noise to be the same for the open-loop measurements as for the residual signal. This is, however, not the case when a feedback law is imposed. It has been shown in the work of Poulsen and Niemann ${ }^{8}$ how to design a residual generator where the noise characteristics are independent of the feedback loop, using active fault detection of a coprime factorised system.

A method for detecting parametric faults by the use of a nominal fault-free model was given in the work of Niemann and Stoustrup. ${ }^{9}$ The method was based on translating different parametric faults to a fault signature system and detecting changes in this fault signature system using a known excitation signal. Furthermore, it was shown in the aforementioned work $^{8}$ how to design a cumulative sum detector for such a problem. Research into linearisation of the fault signature system and isolation of faults have been investigated in the works of Niemann and Poulsen. ${ }^{10,11}$

Detector design for statistical tests has been presented in the work of Kay, ${ }^{12}$ and the Neyman-Pearson detector was presented for optimal detection of partially known signals corrupted with noise. Using a statistical detector has the advantage 
of an easy translation to the false alarm rate and probability of detection, which is the classical success criterion for a fault diagnosis scheme. The detectors introduced in the aforementioned work ${ }^{12}$ assume the noise to be white, which was not the case with the solution presented in the work of Poulsen and Niemann. ${ }^{8}$ A method for isolation of faults based on a sequential detector was proposed in the work of Poulsen and Niemann, ${ }^{13}$ using several excitation signals with different frequencies. An implementation of a statistical detector for detecting faults was proposed first in our other work, ${ }^{14}$ where the fault signature system was linearised. A simulation on how to detect parametric faults on the actuators of an active gas bearing was presented. The linearised fault signature system was however found ill-suited for parametric faults that showed highly nonlinear behaviour. Furthermore, the residual was subject to coloured noise, which resulted in an increase in false alarm occurrences.

In this paper, it is proven possible to implement a detector as presented in the work of Kay ${ }^{12}$ for active fault detection based on the fault signature system. The system to be detected faults on is part of a closed-loop scheme with a known controller. Most systems to be conducted fault detection on are believed to be part of a closed-loop scheme. Prewhitening of the noise has been conducted to improve the results. It is proposed to use the knowledge of the detectors' sensitivity towards different faults to enable isolation of several faults using only a single excitation signal with a known amplitude and frequency. The method presented exploits knowledge about the phase shift and gain impacts of the faults to isolate different faults using only a single signal. Basing the fault diagnosis on the magnitude and phase information makes the solution advantageous compared with other methods such as those previously shown in other works. ${ }^{4-6,15}$ It is shown that it is possible to design the detector based solely on the stochastic model of the noise in addition to the model of the controller and plant. The detector is implemented as a moving window to be able to detect changes over time. The impact of model uncertainties is furthermore discussed. It is believed that a design procedure for residual signals of closed-loop systems that ensures the noise on the residual signal to be white is useful for fault diagnosis of closed-loop systems.

This paper is structured as follows. Section 2 introduces the reader briefly to the preliminary work conducted. Section 3 presents the residual signal design. Section 4 presents the detector design and isolation procedure. Section 6 shows some examples using the method described. This paper is closed with a conclusion in Section 7.

\section{2 | PRELIMINARY RESULTS}

Most detector designs are based on the assumption that the detector signal is influenced by white noise. Such assumptions are usually valid given open-loop problems such as those depicted in Figure 1. Here, plant $G(\theta)$ is subject to a known input $u$, and the output of plant $y_{m}$ is subject to an unknown disturbance $d$, which is in this paper, assumed to be white Gaussian noise.

Such a system structure as presented in Figure 1 is only feasible to use when feedback control is not required, and the plant is open-loop stable. However most interesting control applications require some form of feedback control. When feedback control is applied, the system representation changes to Figure 2, which is the standard implementation used throughout this paper.

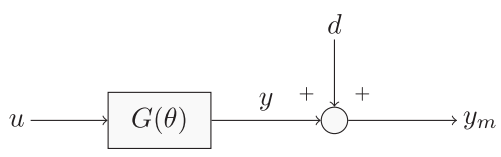

FIGURE 1 Simple open-loop plant setup. Plant $G(\theta)$ is only subject to a known input and the output signal $\left(y_{m}\right)$ is generated by the plant and the unknown disturbance $d$

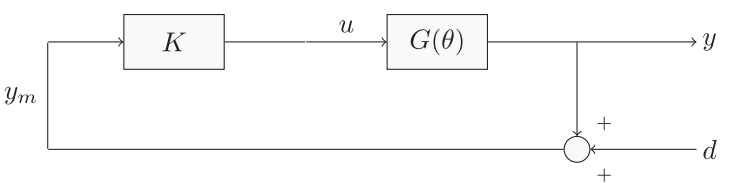

FIGURE 2 Representation of the system description used throughout this paper. The system is here subject to feedback control through the controller $K$. Again, the output is subject to an unknown disturbance signal $(d)$ 
From Figure 2, it is easy to see that the assumption of white noise on the measurement signal $y_{m}$ does not hold due to the feedback loop. The noise signal $d$ is treated as white with a Gaussian distribution for simplicity in this paper. However, much of the analysis conducted throughout this paper is independent of the noise distribution.

\section{1 | Coprime representation}

In this section, a coprime description of the nominal plant and controller are exploited to represent the parametric faults in the plant through the use of the fault signature matrix. Given a nominal plant $G(0)$ and a controller $K(0)$, the coprime factorisation of plant and controller are given in the following 2 equations, respectively.

$$
\begin{array}{ll}
G(0)=N M^{-1}=\tilde{M}^{-1} \tilde{N}, & N, M, \tilde{M}, \tilde{N} \in \mathcal{R} \mathcal{H}_{\infty} \\
K(0)=U V^{-1}=\tilde{V}^{-1} \tilde{U}, & U, V, \tilde{V}, \tilde{U} \in \mathcal{R} \mathcal{H}_{\infty}
\end{array}
$$

Here, the $\sim$ represent the left coprime factorisation, whereas the systems without such are the right coprime factorisation. Such left and right coprime factorisations are always possible to find when the plant and controller can be represented as rational functions. ${ }^{16}$ Given the 8 matrices in Equations (1) and (2), the double Bezout identity shown in Equation (3) is satisfied due to the coprimeness.

$$
\left[\begin{array}{ll}
I & 0 \\
0 & I
\end{array}\right]=\left[\begin{array}{ll}
M & U \\
N & V
\end{array}\right]\left[\begin{array}{cc}
\tilde{V} & -\tilde{U} \\
-\tilde{N} & \tilde{M}
\end{array}\right]=\left[\begin{array}{cc}
\tilde{V} & -\tilde{U} \\
-\tilde{N} & \tilde{M}
\end{array}\right]\left[\begin{array}{ll}
M & U \\
N & V
\end{array}\right]
$$

Given a nominal plant as defined in Equation (1), it is possible to define all stabilising controllers based on a nominal stabilising controller. Such a controller is given in Equation (4) using the right coprime factorisation and in Equation (5) based on the left coprime factorisation of plant and controller. ${ }^{17}$

$$
\begin{aligned}
& K(Q)=(U+M Q)(V+N Q)^{-1}, \quad Q \in \mathcal{R} \mathcal{H}_{\infty} \\
& K(Q)=(\tilde{V}+Q \tilde{N})^{-1}(\tilde{U}+Q \tilde{M}), \quad Q \in \mathcal{R} \mathcal{H}_{\infty}
\end{aligned}
$$

$K(Q)$ can be represented as a linear fractional transformation (LFT). A lower LFT of the parametrised controller $K(Q)$ is given by

$$
\begin{aligned}
K(Q) & =\mathcal{F}_{l}\left(J_{K}, Q\right) \\
J_{K} & =\left[\begin{array}{cc}
U V^{-1} & \tilde{V}^{-1} \\
V^{-1} & -V^{-1} N
\end{array}\right] .
\end{aligned}
$$

In principle, it is possible to design a realisation as in Equation (6) of any linear controller. Such a control implementation is shown in Figure 3. The system shown in Figure 3 is the same as presented in Figure 2; however, 2 new signals $\alpha$ and $\beta$ are introduced to the closed-loop system description.

It is shown in the work of Tay et $\mathrm{al}^{17}$ how to transform some of the most general controller schemes into the form shown in Figure 3.

It is possible to parametrise all plants stabilised by the nominal controller. In Equations (8) and (9), a parametrisation of all plants stabilised by a nominal controller based on the right and left coprime factorisation are respectively given.

$$
\begin{aligned}
& G(S)=(N+V S)(M+U S)^{-1}, \quad S \in \mathcal{R} \mathcal{H}_{\infty} \\
& G(S)=(\tilde{\mathrm{N}}+S \tilde{U})^{-1}(\tilde{M}+S \tilde{V}), \quad S \in \mathcal{R} \mathcal{H}_{\infty}
\end{aligned}
$$

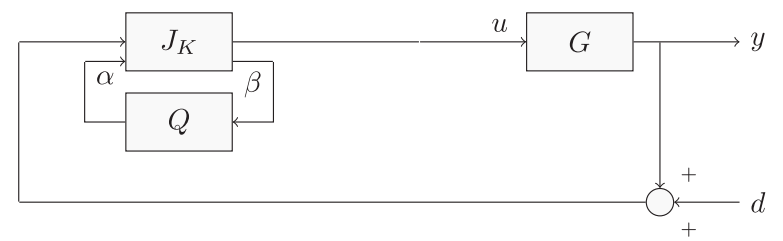

FIGURE 3 Closed-loop system setup using the Youla parametrisation of the controller 
Equivalently, as for the parametrised controller, it is possible to represent the parametrised plant as an LFT

$$
\begin{aligned}
G(S) & =\mathcal{F}_{u}\left(J_{G}, S\right) \\
J_{G} & =\left[\begin{array}{lr}
-M^{-1} U & M^{-1} \\
\tilde{M}^{-1} & N M^{-1}
\end{array}\right] .
\end{aligned}
$$

A block diagram of the closed-loop system introduced in Figure 2 with the parametrised plant using Equation (10) is shown in Figure 4.

Again, 2 new signals are introduced, ie, $\eta$ and $\epsilon$. The transfer functions $S$ parametrising all plants stabilised by the nominal controller have been known as the open-loop error between the nominal and real plant ${ }^{18}$ and describe the dynamics of the true system omitted from the nominal model. It was shown in the work of Anderson ${ }^{18}$ that the signal $\eta$ is independent of the output noise $d$.

The signals $\eta$ and $\epsilon$ are, however, not directly measurable. It was shown in the aforementioned work ${ }^{17}$ that the relationship between the set of signals $\{\alpha, \beta\}$ from Figure 3 and the set of signals $\{\eta, \epsilon\}$ from Figure 4 can be depicted as in Figure 5.

There are several ways to prove the relationship between the 2 set of signals $\{\alpha, \beta\}$ and $\{\eta, \epsilon\}$, with one approach to be found in our other work. ${ }^{19}$ Whereas, it is not possible to directly excite the system using $\eta$, it is using the signal $\alpha$. It is therefore convenient to use the signals related to the controller for identification and detection purposes.

The system setup in Figure 6 is equivalent to the setup shown in Figure 3 but with $Q$ omitted. Furthermore, it is also straightforward to see from Figure 6 that the controller is not changed from the nominal one as long as $Q$ is disconnected.

In order to state the detection problem, the closed-loop system with regard to the external inputs is required. The transfer functions are shown in Equation (12) and are derived from Figure 4 with the input and output vector defined as in Equation (12), as well as the connection of the signals shown in Figure 5.

$$
\left[\begin{array}{l}
y \\
u \\
\beta
\end{array}\right]=\left[\begin{array}{cc}
(N+V S) \tilde{U} & N+V S \\
(M+U S) \tilde{U} & M+U S \\
\tilde{M}+S \tilde{U} & S
\end{array}\right]\left[\begin{array}{l}
d \\
\alpha
\end{array}\right]=P_{c l}(S)\left[\begin{array}{l}
d \\
\alpha
\end{array}\right]
$$

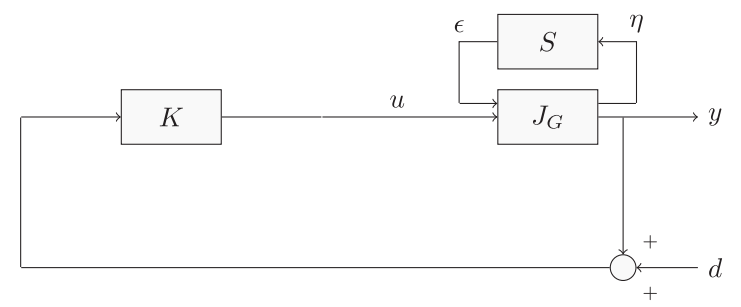

FIGURE 4 Closed-loop system setup using the Youla parametrisation of the plant

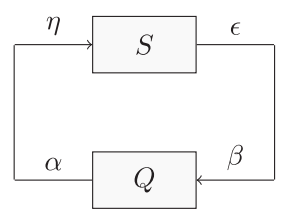

FIGURE 5 Figure showing the relationship between the input-output pair of $S$ and $Q$

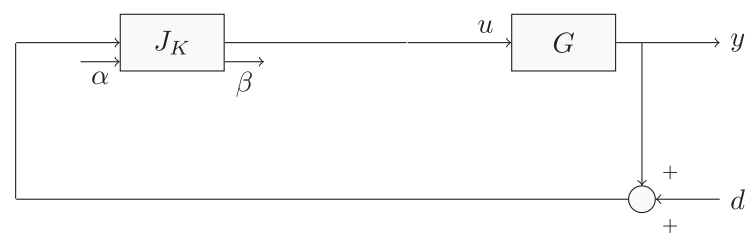

FIGURE 6 Alternative controller representation based on a coprime factorisation of the controller and plant model 
Here, the $\beta$ output is considered the detection or residual signal. The input signal $d$ is considered an unknown input, whereas $\alpha$ is the controllable input used for active fault detection.

\section{2 | Fault diagnosis in the YJBK setup}

In order to detect faults in the system based on the fault signature system $S$, it is necessary to translate the behaviour of parametric faults from the input-output pair $u, y$ to the signal pair $\eta, \epsilon$. Such a parametrisation was first introduced in the work of Youla et al, ${ }^{20}$ and the methods to represent different parametric faults were first presented in the work of Niemann. ${ }^{21}$ This method is based on a coprime factorisation of a nominal model of the implemented plant and controller to design a residual signal, which is zero for the fault-free case and nonzero when a fault occurs. However, to define the parametric faults through the fault signature system, it is first necessary to establish how faults on the plant are represented. Given a parametric fault $\theta$ assumes that the plant can be defined as an upper LFT, as shown in Equation (13), where $G_{z w}, G_{z u}, G_{y w}$, and $G_{y u}$ are defined using only the fault-free plant and the parametric fault $\theta . \theta$ is given as a diagonal matrix where each diagonal element, denoted $\theta_{i}$, corresponds with a parametric fault.

$$
G(\theta)=\mathcal{F}_{u}\left(\left[\begin{array}{ll}
G_{z w} & G_{z u} \\
G_{y w} & G_{y u}
\end{array}\right], \theta\right)
$$

Instead of expressing plant $G$ as a function of $\theta$, it is advantageous to formulate the fault signature system as a function of the unknown parametric faults $\theta$. Given faults in the form presented in Equation (13), it was shown in the work of Niemann and Poulsen ${ }^{10}$ that the fault signature system can be expressed as

$$
S(\theta)=\mathcal{F}_{l}\left(\left[\begin{array}{cc}
0 & \tilde{M} G_{y w} \\
G_{z u} M & G_{z w}+G_{z u} U \tilde{M} G_{y w}
\end{array}\right], \theta\right) .
$$

It is clear from Equation (14) that the fault signature system $S(\theta)$ is a nonlinear function with regard to the parametric faults. The gain and the phase shift of the fault signature system depend on the parametric fault and are, therefore, of interest for fault diagnosis. With the fault signature system given with respect to the parametric faults, it is useful to state the diagnosis problem solved in this paper.

The main advantage of using the fault signature system as described in Equation (14) comes from the fact that $S(\theta)$ is zero in the fault-free case, ie,

$$
S(0)=0
$$

While it is different from zero when a detectable fault occurs, ie,

$$
S(\theta) \neq 0 \quad \forall \quad \theta \neq 0 .
$$

The effect of faults on $S$ is thus similar to the effect of faults on the residual generator used for passive fault detection.

\section{3 | DETECTION PROBLEM FORMULATION}

In Section 2.2, the connection between parametric faults and the fault signature system was established based on the system description given in Section 2.1. In this section, the residual signal used for detection is presented, and the assumptions used for detector design in the following sections are presented.

Using Equation (12), the residual signal is

$$
\beta[n]=S(\theta) \alpha[n]+(\tilde{M}+S(\theta) \tilde{U}) d[n] .
$$

The residual in Equation (17) is influenced by white noise $d$, which goes through a filter. The residual signal $\beta$ is therefore influenced by coloured noise. In active fault diagnosis, the goal of the detector is to detect a known signal with unknown amplitude and phase, corrupted by noise, compactly written

$$
\beta[n]=A_{S_{\theta}} \alpha\left[n-n_{0}\right]+(\tilde{M}+S(\theta) \tilde{U}) d[n] \quad n=0,1, \ldots ., N-1 .
$$


Here, $\alpha[n]$ is the known excitation signal at sample $n, A_{S_{\theta}}$ is the gain through the fault signature system of the known excitation signal $\alpha[n], n_{0}$ is the delay of the excitation signal through the fault signature system, and $d[n]$ is the noise signal, which in this paper is assumed to be white Gaussian noise. For the transition from Equations (17) to (18), it is assumed that the excitation signal $\alpha$ is having a period sufficiently long compared with the sampling period. Furthermore, the period of $\alpha$ is possible to describe as a whole multiple of the sampling period.

For the detection methods presented in this paper, the noise is modelled as Gaussian. However, the noise is not required to be Gaussian to design a matched filter detector, but this requirement is maintained throughout this paper for simplicity. Whiteness of the noise is, on the other hand, a strict requirement to be able to give an upper bound to the number of false alarms in the fault-free case. Given the method presented, the noise on the residual signal is kept white in the fault-free case when the system is only subject to white noise. Furthermore, it is mentioned briefly how to keep the whiteness requirement for a system subject to both system and measurement noise.

\section{1 | Design of whitening filter}

In the first case for a signal as given in Equation (18) and letting $\mathcal{H}_{0}$ be the fault-free case, since $S(0)$ is zero, the residual signal $\beta$ simplifies to

$$
\mathcal{H}_{0}: \beta[n]=\tilde{M} d[n] .
$$

Since $\tilde{M}$ is a model-dependent known system, the residual signal is redefined to

$$
\mathcal{H}_{0}: r[n]=\tilde{M}^{-1} \beta[n]=d[n] .
$$

It is clear from Equation (20) that if the noise $d$ is white Gaussian, then the noise imposed on the residual signal in Equation (20) is white Gaussian as well. Furthermore, the variance of the noise on the residual signal is equivalent to the variance of the measurement noise. Designing the decorrelation filter $\tilde{M}^{-1}$ is only feasible for open-loop stable systems. Given any open-loop unstable system $\tilde{M}^{-1}$ will be unstable, and instead, a stable filter with the same correlation as $\tilde{M}^{-1}$ is required.

Such a filter can be realised by a spectral factorisation, finding a stable filter with the same prewhitening effect as the unstable filter $\tilde{M}^{-1}$. In this paper, all theory is handled in discrete time; however, a continuous equivalent is also possible by mirroring the right half-plane zeros through zero on the real axis.

For the system $\tilde{M}$ containing zeros outside the stability area, it is possible to design a system, which has no zeros outside the stable area with the same spectrum denoted $W$. The method can be found in the work of Gustafsson ${ }^{22}$ and a transformation that keeps the same spectrum is shown in Equation (21) while transforming the unstable solution into a stable one.

$$
W(\bar{z}) W^{T}(z)=\tilde{M}(\bar{z}) \tilde{M}^{T}(z)
$$

The stable filter is thus denoted $W^{-1}$, and the detection signal is given in Equation (22) with the proposed stable filter that makes the noise white on the detector signal in the fault-free case, ie,

$$
\mathcal{H}_{0}: r[n]=W^{-1} \beta[n]=H d[n],
$$

where $H=W^{-1} \tilde{M}$ is a constant diagonal matrix..$^{22}$ Since $H$ contains no dynamics the detector signal is again only subject to white Gaussian noise; however, the method does alter the variance of the noise, as shown by the constant $H$ matrix.

The methods presented above are based on the assumption that all noise can be modelled as white Gaussian noise on the output. For the case of a white Gaussian process and measurement noise, designing a Kalman filter is proposed instead. A Kalman filter ensures that the noise is white on the innovation signal and thereby on $\beta$, as shown in the work of Niemann. ${ }^{23}$

In this paper, the detectors are designed based on white Gaussian output noise only. Therefore, if the system is open-loop unstable, the residual is given by Equation (23), where the variance of the noise $d$ in the fault-free case has been subject to the constant diagonal matrix $H$.

$$
r=W^{-1} \tilde{M} G_{y w} \theta\left(I-\left(G_{z w}+G_{z u} U \tilde{M} G_{y w}\right) \theta\right)^{-1} G_{z u} M \alpha+\left(H+W^{-1} S(\theta) \tilde{U}\right) d
$$


For the case that the plant is open-loop stable, the residual signal can be simplified. The residual signal of interest to detect in white Gaussian noise is then with regard to the parametric uncertainties given as

$$
r=G_{y w} \theta\left(I-\left(G_{z w}+G_{z u} U \tilde{M} G_{y w}\right) \theta\right)^{-1} G_{z u} M \alpha+\left(I+\tilde{M}^{-1} S(\theta) \tilde{U}\right) d .
$$

\section{4 | FAULT DIAGNOSIS}

In this section, the detector design is presented together with calculations of the probability of detection and the threshold. The matched filter detector with a moving window is chosen since it is natural to design based on a wanted false alarm rate. The detector design is based on the residual signal given by Equation (23), which is the general case. It is assumed that several different faults can occur in the plant; however, multiple faults cannot happen simultaneously. In order to detect the faults, a moving window is used. The detector is based on selecting 1 input and 1 output of the plant to use for diagnosing the faults. This section is structured such that the first detection design is presented without any further assumptions. The ability to isolate faults is then discussed and test statistics for the detector design is given. Finally, the detector design is presented for the case of unique phase shifts for each of the faults considered.

\section{1 | Detector design}

Using the framework introduced in the work of Basseville and Nikiforov ${ }^{24}$ to design the detector a $\mathcal{H}_{0}$ hypothesis is constructed in Equation (25) for the fault-free case, and a $\mathcal{H}_{1}$ hypothesis is constructed in Equation (26) representing the case that one of the possible faults has occurred.

$$
\begin{array}{ll}
\mathcal{H}_{0}: r[n]=H d[n], & n=0,1, \ldots, N-1 \\
\mathcal{H}_{1}: r[n]=A_{S_{\theta}} \alpha\left[n-n_{0}\right]+\left(H+W^{-1} S(\theta) \tilde{U}\right) d[n], & n=0,1, \ldots, N-1
\end{array}
$$

Here, $d$ is white Gaussian noise, and $A_{S_{\theta}}$ is the amplification of the detector signal through the system $W^{-1} S(\theta)$, which both depends on which fault is occurring and the magnitude of the fault. $n_{0}$ is the unknown delay due to the fault occurring and the magnitude of this fault. Only faults causing parameter degradation are considered in this paper, which limits the relative faults $\theta_{i}$ to [ -10 [, where 0 corresponds to no fault and -1 corresponds to a complete failure. There is thus no sign change through the whole possible fault range, which makes a Neyman-Pearson detector feasible. ${ }^{12}$

The design of the detector is based on the $\mathcal{H}_{1}$ hypothesis given a predetermined possible fault. A moving window is used for the detector such that it is possible to determine when a change occurs. Since the delay is unknown and depends on the fault occurring, it is required to determine the delay of detector signal, which can be accomplished using

$$
\phi=\arg \max _{n_{0} \in \tau}\left(\sum_{n=n_{0}}^{n_{0}+N-1} r[n] \alpha\left[n-n_{0}\right]\right) .
$$

Here, $\phi$ is the maximum-likelihood estimate of the delay, $\tau$ is the set of possible delays given a priori knowledge about the parametric faults, and $N$ is the window length. With the delay estimated using Equation (27), the test statistic can be designed. For the generalised case, the test statistic is

$$
T(r)=\sum_{n=\phi}^{\phi+N-1} r[n] A_{0} \alpha[n-\phi] .
$$

Here, $A_{0}$ is a predetermined amplification of the signal excitation $\alpha$ through the fault signature system given the initial guess of the magnitude of one of the faults. It is possible to design other test statistics, which might be beneficial in special cases.

An example of such a test statistic is given in Section 4.4, which for certain assumptions has attributes that simplify the test statistics.

In fault detector design, the probability of a false alarm and probability of detection are usually the 2 design criteria. For a detector as described in Equation (28), the probability of false alarm is used to determine the threshold $\gamma$, as shown 
in Equation (29), ie,

$$
\begin{aligned}
& \gamma=\sqrt{\sigma^{2} \nu} Q^{-1}\left(P_{F A}\right) \\
& \nu=N \frac{A_{0}^{2}}{2} .
\end{aligned}
$$

Here, $\sigma^{2}$ is the variance of the noise, $v$ is the energy contained in the signal for one window length given a sinusoidal wave, $Q(\cdot)$ is the complementary cumulative distribution, and $P_{F A}$ is the allowed frequency of false alarms. It is worth noticing that the threshold is independent of the different possible faults. The $\mathcal{H}_{1}$ hypothesis chosen by Equation (27) is discarded if $T(r)$ is below the threshold $\gamma$ and found to be true if $T(r)$ is above the threshold $\gamma$.

With a threshold design based on the allowed probability of a false alarm, the probability of detection is

$$
P_{D}=Q\left(Q^{-1}\left(P_{F A}\right)-\sqrt{D}\right) .
$$

The probability of detection is based on the assumption of the noise to be white, which it is not in the case when $\mathcal{H}_{1}$ is true. The variance of the detector signal will, therefore, be higher than approximated. The deflection coefficient $D$ is

$$
D^{2}=\frac{\left(E\left(T ;\left.\mathcal{H}_{1}\right|_{\theta_{k}}\right)-\mathcal{X}\right)\left(E\left(T ;\left.\mathcal{H}_{1}\right|_{\theta_{A_{0}}}\right)-\mathcal{X}\right)}{\operatorname{var}\left(T ; \mathcal{H}_{0}\right)} .
$$

The deflection coefficient depends on the fault detected $\theta_{k}$ and the initial guess of the fault $\theta_{A_{0}}$. It is therefore necessary to calculate the probability of detection separately for each fault considered. It is worth noticing that Equation (32) is only true when the test statistic in Equation (28) is used.

Here, $\mathcal{X}$ is defined in Equation (33) and is the expected value of the test statistic given that the $\mathcal{H}_{0}$ is true.

$$
\mathcal{X}=E\left(T ; \mathcal{H}_{0}\right)
$$

Using Equation (31), it is possible to determine the smallest parametric fault with an acceptable probability of detection given the desired probability of the false alarm rate.

\section{2 | Design of the excitation signal}

It is of paramount importance to be able to design the excitation signal in accordance with predetermined criteria. In this section, one such criterion is presented so that it is possible to design the excitation signal. It is for simplicity decided to use only one input for the excitation signal and one output of the fault signature system. The relevant fault signature system thus becomes a SISO system, which makes the analysis simpler. In this paper, the shape of the excitation signal has been limited to be a single sinusoidal wave with the single degree of freedom being the frequency. It is the task of the excitation signal to maximise the impact of a fault in the residual signal. Thus, it is desired to determine the gain of the transfer function from the excitation signal to the residual given as

$$
\xi_{r}(\omega)=\left|W^{-1} \tilde{M} G_{y w} \theta\left(I-\left(G_{z w}+G_{z u} U \tilde{M} G_{y w}\right) \theta\right)^{-1} G_{z u} M\right| .
$$

If the design criteria is simply to maximise the amplitude of the excitation signal, the goal is to maximise Equation (34). However, it is believed that for most systems the design of the excitation signal should be a trade-off between maximising the signature of faults while minimising the impact of the excitation signal on the system outputs. The highest gain from the excitation signal to the system outputs can be found using Equation (12) and is

$$
\xi_{y}(\omega)=\bar{\sigma}(N(\omega))
$$

Here, $\bar{\sigma}$ denotes the highest singular value, ${ }^{25}$ which corresponds to the direction with the highest gain for a MIMO system. It is possible to define a criteria for the design of the excitation signal with the gain from the excitation signal to the residual and outputs respectively determined as

$$
f_{\alpha}(\omega)=\frac{\xi_{r}(\omega)}{\xi_{y}(\omega)} .
$$


The frequency of the excitation signal is thus chosen.

$$
\omega_{\alpha}=\underset{\omega_{1} \leq \omega \leq \omega_{2}}{\operatorname{argmax}} f_{\alpha}(\omega)
$$

Here, $\omega_{1}$ defines the lowest considered frequency, and $\omega_{2}$ is the highest frequency considered for the sinusoidal excitation signal. For many systems, the range of possible frequencies might be limited due to mechanical limitations or the sample speed, which would naturally define the upper and lower limit. So far, only a single fault is considered for detection. For several possible faults, the frequency should be chosen such that the detectability of the fault most difficult to detect is maximised why Equation (36) is changed to

$$
f_{\alpha}(\omega)=\min _{\theta=\Delta} \frac{\xi_{r}(\theta, \omega)}{\xi_{y}(\omega)} .
$$

Here, $\Delta$ denotes the set of different possible faults considered. With Equation (36) reformulated as Equation (38), the optimal excitation frequency can again be found using (37).

\section{3 | Isolation of faults}

In this section, the ability to isolate faults is discussed. The discussion is based on an excitation signal containing a single sinusoidal wave and estimating the phase shift using Equation (27). It is in the general case that it is not possible to isolate a fault only based on the phase shift, given that the phase shifts of several faults might be the same. An example is given in Figure 7 on how 2 parametric faults share the same phase shift.

It is shown in Figure 7 that the set of possible phase shift for fault 1 is a subset of the set of possible phase shifts for the second parametric fault. It is therefore not possible to distinguish when fault 1 has occurred from the instances of fault 2 occurring solely based on the phase shift. It is thus seen to be convenient to exploit the knowledge about the phase shift and the gain of the fault signature system. Using the test statistic $T(r)$ from Equation (28) with the moving window $N$, which are whole periods, the energy of the signal is

$$
\kappa=N \frac{A_{k} A_{0}}{2} .
$$

Here, $A_{k}$ is the amplitude of the sinusoidal wave $r[n]$. Using the fact the noise is Gaussian with a mean of 0 , by isolating $A_{k}$ in Equation (39), the amplitude of the sinusoidal detector wave can be found as Equation (40) for an appropriate

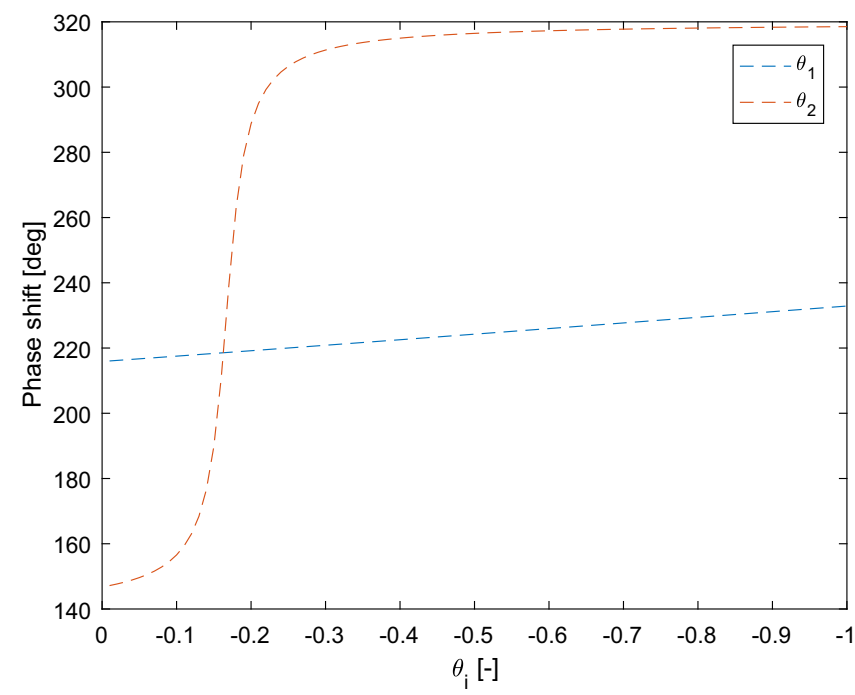

FIGURE 7 Phase shift of a sinusoidal wave for an example system with 2 possible parametric faults. Here, it is seen that all delays that could correspond to parametric fault $1\left(\theta_{1}\right)$ occurring might as well correspond to parametric fault $2\left(\theta_{2}\right)$ occurring with a magnitude degradation around $20 \%$ [Colour figure can be viewed at wileyonlinelibrary.com] 


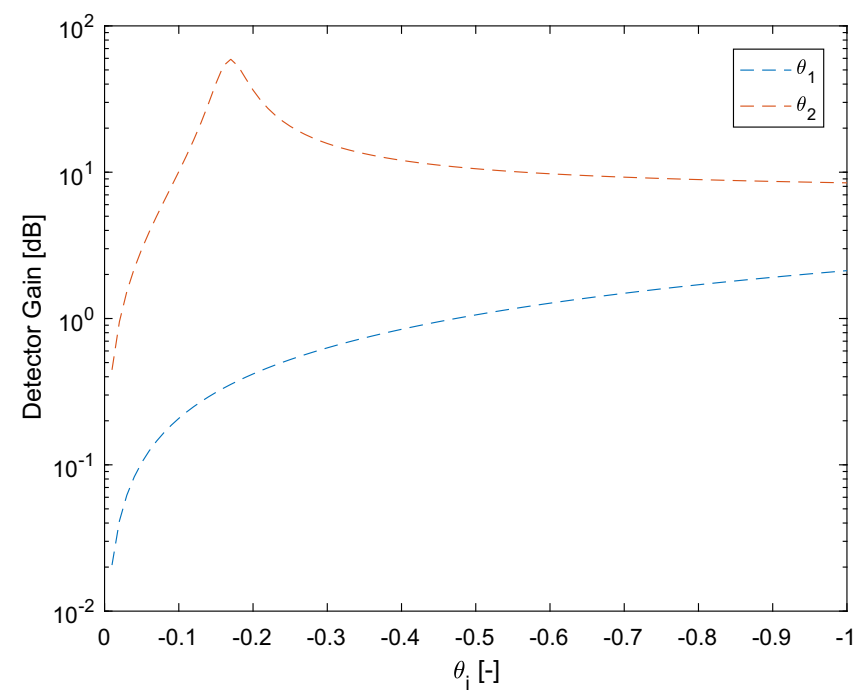

FIGURE 8 Gain of a sinusoidal wave for an example system with 2 possible parametric faults [Colour figure can be viewed at wileyonlinelibrary.com]

window length.

$$
A_{k}=\frac{2 \kappa}{N A_{0}}
$$

The gain from the excitation signal to the detection signal is thus chosen.

$$
A_{S_{\theta}}=\frac{A_{k}}{|\alpha|}
$$

As with the phase shift, it is also possible to determine how the gain of the excitation signal changes with regard to the magnitude of the fault for a specific frequency of the sinusoidal signal.

The gain of the excitation signal is shown in Figure 8 for the same system and same parametric faults as were presented in Figure 7. By combining the two, it is easy to see that it is possible to isolate which fault has occurred. The fault isolation so far is based on choosing the frequency that makes the job of fault detection easiest. Such a choice does not consider whether the fault characteristics are similar or not and might therefore be a poor choice when it comes to fault isolation. In such a case, it can be beneficial to change the frequency of the sinusoidal wave of the excitation signal to maximise the difference between the fault signatures when a fault is detected and isolate the fault through a second test.

\section{4 | Multiple faults without phase shift crossing}

The method presented in Section 4.1 might for some cases be simplified. Depending on how the phase shift develops if there is a unique mapping between the phase shift and which fault occurs a simplification is possible. This corresponds to the phase shift lines in Figure 7 not reaching the same vertical level at any point. If such a mapping is possible, the test statistic can be expressed as

$$
T(r)=\sum_{n=\phi}^{\phi+N-1} r[n] A_{\phi} \alpha[n-\phi] .
$$

Here, $A_{\phi}$ is the gain from the excitation signal $\alpha$ to the residual signal $r$ based on the fault determined by the delay using Equation (27). The gain thus changes with regard to the delay identified, which results in a change of the test statistic. Besides changing the test statistic the detector used in Equation (42) has the advantage of directly identifying which fault is occurring. The threshold is again given as in Equation (43), but the energy of the signal is now determined by Equation (44).

$$
\begin{aligned}
& \gamma=\sqrt{\sigma^{2} \nu} Q^{-1}\left(P_{F A}\right) \\
& \nu=N \frac{A_{\phi}^{2}}{2}
\end{aligned}
$$


With the threshold determined from Equation (43), the probability of detection is

$$
P_{D}=Q\left(Q^{-1}\left(P_{F A}\right)-\sqrt{D}\right) \text {. }
$$

The probability of detection is thus given in the same way for both detectors. The difference is a bit subtle and comes in the change of the definition of the deflection coefficient $D$. Here, $D$ is

$$
D^{2}=\frac{\left(E\left(T ;\left.\mathcal{H}_{1}\right|_{\theta_{k}}\right)-E\left(T ; \mathcal{H}_{0}\right)\right)^{2}}{\operatorname{var}\left(T ; \mathcal{H}_{0}\right)}
$$

The deflection coefficient depends on the fault detected $\theta_{k}$ and must be calculated separately for each fault considered. It is worth noticing that Equation (46) is only true when the test statistic in Equation (42) is used, and the deflection coefficient is much more intricate when the assumption of no phase shift crossing cannot be used.

\section{5 | ANALYSIS OF MODEL UNCERTAINTIES}

So far, a perfect model without any uncertainties has been assumed. It is however important to examine the impact of model uncertainties on the residual signals derived. For this analysis, only parametric uncertainties are considered, and the impact of higher-order dynamics not contained in the model are disregarded. For parametric uncertainties $\mu$, the residual signal is expressed in Equation (47).

$$
r=W^{-1} S(\theta, \mu) \alpha+\left(H+W^{-1} S(\theta, \mu) \tilde{U}\right) d
$$

The $\mathcal{H}_{0}$ when no fault occurs is then given in Equation (48), whereas the $\mathcal{H}_{1}$ is given in Equation (49).

$$
\begin{aligned}
& \mathcal{H}_{0}: r[n]=A_{S_{\mu}} \alpha[n]+H d[n] \\
& \mathcal{H}_{1}: r[n]=A_{S_{(\theta, \mu)}} \alpha\left[n-n_{0}\right]+\left(H+W^{-1} S(\theta, \mu) \tilde{U}\right) d[n]
\end{aligned}
$$

Here, $A_{S_{\mu}}$ is the gain through $W^{-1} S(\mu)$ given the frequency of the signal $\alpha . \mu$ defines the bound on the uncertainty set; however, since the open-loop error is nonlinear with regard to the parametric uncertainties, the highest amplitude of $A_{S_{\mu}}$ might not be at the boundary. A search in the uncertainty space can thus be conducted to find the uncertainty that maximises the gain from the excitation to the residual signal. Such a problem is an $N$-dimensional problem, where $N$ is the number of parametric uncertainties. It might therefore be convenient to linearise $W^{-1} S(\mu)$ and determine the gain from the uncertainty bound if the dimension is too large. The threshold is therefore given similarly as the threshold for the case of no uncertainties with the addition of a bias, which depends on the uncertainty bound, as shown in Equation (50).

$$
\begin{aligned}
\gamma^{\prime}=\gamma+v_{\mu} & =\sqrt{\sigma^{2} v} Q^{-1}\left(P_{F A}\right)+v_{\mu} \\
v_{\mu} & =N \frac{\left(A_{0} A_{S_{\mu}}\right)^{2}}{2}
\end{aligned}
$$

Here, $A_{0}$ is replaced with $A_{\phi}$ if it is possible to use the detector presented in Equation (42), used for the case when no phase shift crossing is present. Furthermore, $A_{S_{\mu}}$ is the maximal amplitude of the signal $r$ due to the uncertainty such that the constant $v_{\mu}$ is the energy of the detector due to the uncertainty and is an upper bound. The threshold is therefore determined such that the number of false alarms never exceeds the allowed amount. The threshold given in Equation (50), however, is only valid when Equation (52) is true.

$$
\bar{\sigma}(H) \gg \bar{\sigma}\left(W^{-1} S(\theta, \mu) \tilde{U}\right)
$$

If Equation (52) is true, the noise can be seen as approximately white and Gaussian in the fault-free case. Such an assumption as given in Equation (52) is usually valid when proper identification of the system has been conducted beforehand. 


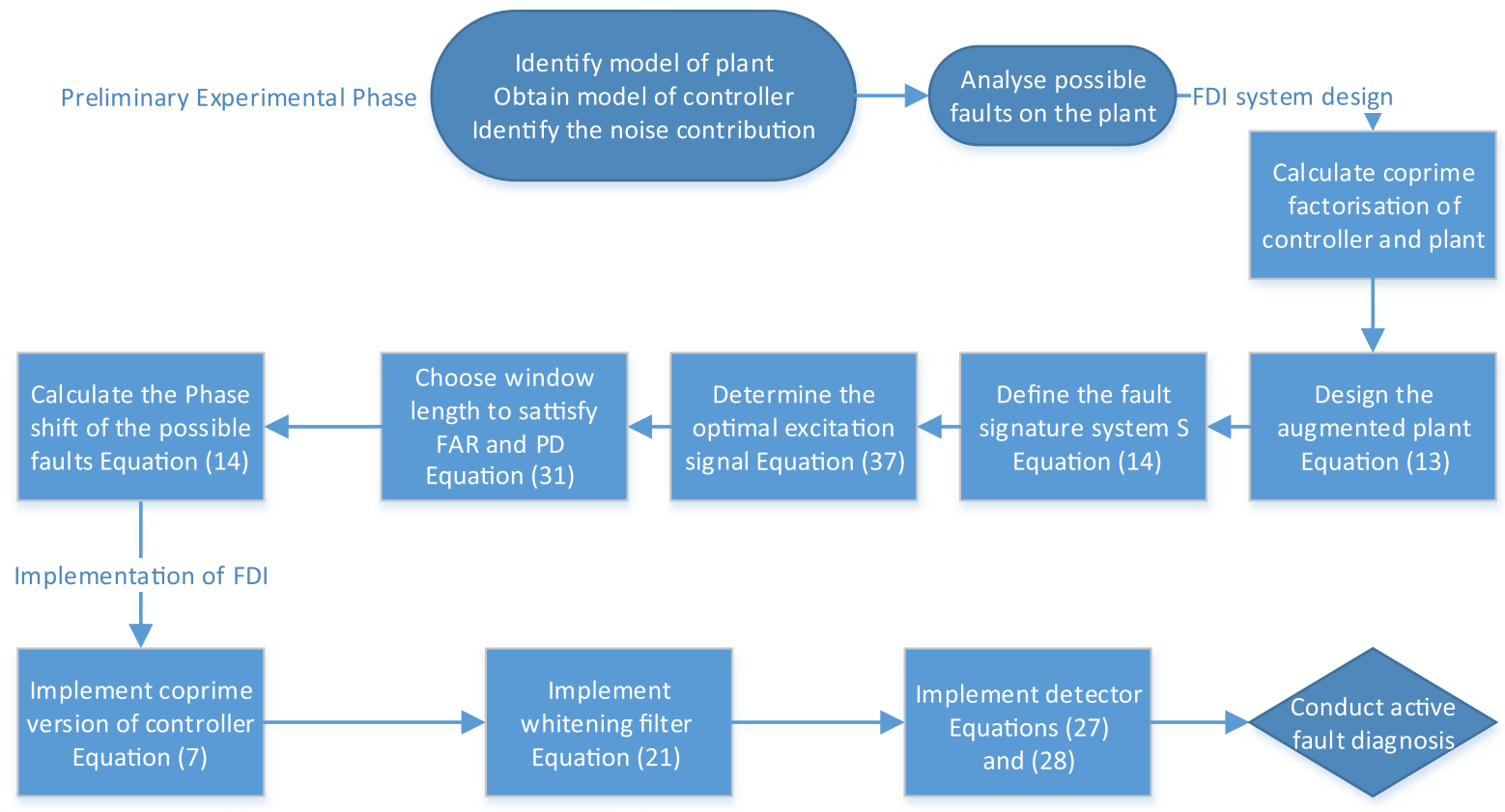

FIGURE 9 Flow chart of the steps involved in using the active fault detection and isolation (FDI) method. The round box symbolise information that need to be obtained, the rectangular box symbolise steps derived using already known information and the pointy box symbolise actions [Colour figure can be viewed at wileyonlinelibrary.com]

\section{1 | Summary of method}

The theory has been outlined in the previous sections for how to design a matched filter detector for active fault detection and isolation. The steps to go through for applying the active fault detection and isolation method is shown in Figure 9. The flow chart is divided into 3 steps: preliminary experimental work, design phase, and implementation.

The method was shown to be based a model of the plant, controller, and considered faults. Furthermore, knowledge about the PDF and the correlation of the noise is required. Based on this initial information, it is was shown possible to design a detector based on a wanted false alarm rate.

\section{6 | EXAMPLES}

In this section, examples are given to introduce the reader to the method. Simulation examples are given on how to apply the method given in this paper. A discrete second-order SISO state space system is used to show how a parametric change can be detected. A MIMO system could as easily be used for the fault detection but is excluded here for simplicity. White Gaussian output noise with a variance of 0.11 is applied to the system. The sample time is $1 \mathrm{~ms}$, and the 4 matrices of the discrete state space system are given in Equations (53) to (56).

$$
\begin{aligned}
A & =\left[\begin{array}{ll}
a_{11} & a_{12} \\
a_{21} & a_{22}
\end{array}\right]=\left[\begin{array}{cc}
1 & -0.3 \\
-0.5 & 0.2
\end{array}\right] \\
B & =\left[\begin{array}{l}
b_{1} \\
b_{2}
\end{array}\right]=\left[\begin{array}{l}
2 \\
3
\end{array}\right] \\
C & =\left[\begin{array}{ll}
c_{1} & c_{2}
\end{array}\right]=\left[\begin{array}{ll}
-1 & 4
\end{array}\right] \\
D & =[0]
\end{aligned}
$$

The state space system used for the example here has a pole outside the unit circle, and it is therefore an open-loop unstable system. In order to stabilise the system, an observer is designed with a linear quadratic regulator state feedback and observer gain. Furthermore, the input gains $b_{1}$ and $b_{2}$ are prone to multiplicative faults. The input matrix is therefore 
redefined as in Equation (57).

$$
B=\left[\begin{array}{l}
b_{1}\left(1+\theta_{1}\right) \\
b_{2}\left(1+\theta_{2}\right)
\end{array}\right]
$$

The augmented system taking the faults into account is given in Equation (58) using the system description presented in Equation (13).

$$
\begin{aligned}
{\left[\begin{array}{l}
z \\
y
\end{array}\right] } & =\left[\begin{array}{cc|ccc}
a_{11} & a_{12} & b_{1} & 0 & b_{1} \\
a_{21} & a_{22} & 0 & b_{2} & b_{2} \\
\hline 0 & 0 & 0 & 0 & 1 \\
0 & 0 & 0 & 0 & 1 \\
c_{1} & c_{2} & 0 & 0 & 0
\end{array}\right]\left[\begin{array}{l}
w \\
u
\end{array}\right] \\
w & =\left[\begin{array}{cc}
\theta_{1} & 0 \\
0 & \theta_{2}
\end{array}\right] z
\end{aligned}
$$

The frequency of the excitation signal was chosen based on the method described in Section 4.2. Using Equations (37) and (38), the lower bound $\omega_{1}$ is chosen to be $10 \mathrm{rad} / \mathrm{s}$. The upper bound $\omega_{2}$ is chosen to be $125 \mathrm{rad} / \mathrm{s}$ such that the sampling time is still sufficiently higher.

$$
\omega_{\alpha}=\underset{10 \leq \omega \leq 125}{\operatorname{argmax}} f_{\alpha}(\omega)=125
$$

Using Equation (60), the frequency of the excitation signal is chosen to be $125 \mathrm{rad} / \mathrm{s}$. An illustrative plot of the efficiency for detecting each of the 2 possible faults using Equation (36) is shown in Figure 10, where $\theta_{1}$ and $\theta_{2}$ equal to -0.1 have been considered, respectively.

Using Figure 10, it is easy to see that the fault that is most difficult to detect is easier to detect as the frequency of the excitation signal is increased. With the augmented system description presented in Equation (58) and the Phase shift of a sinusoidal wave of $125 \mathrm{rad} / \mathrm{s}$ introduced as the $\alpha$ signal upon the residual signal $r$. The blue dashed line corresponds to $\theta_{1}$, and the red dashed line corresponds to $\theta_{2}$. excitation signal chosen to be a sinusoidal wave of $125 \mathrm{rad} / \mathrm{s}$, it is possible to determine the relationship between the phase shift and the magnitude of the fault occurring. Due to the pole location outside the unit circle, the detector signal is given by Equation (22). The phase shift with regard to the magnitude of the fault is thus, as shown in Figure 11.

Before designing the detector, it is important to verify that the noise is white on the residual signal. It is possible to verify the whiteness of the residual signal by looking at the autocorrelation of the signal in the fault-free case.

The autocorrelation of the innovation signal and the innovation signal with the whitening filter $W$ applied are presented in Figure 12. The filter $W$ is used since the system itself contains an unstable pole outside the unit circle, and $\tilde{M}^{-1}$ is, thus, not stable. From Figure 12, it is seen that the noise is white after applying the whitening filter $W$, and thus, a detector can be designed. Furthermore, by measuring the residual signal in the fault-free case, the variance of the noise was found to be 0.14 on the residual signal and $A_{0}$ was decided to be a $10 \%$ fault on $\theta_{1}$, which is equal to the fault introduced in the

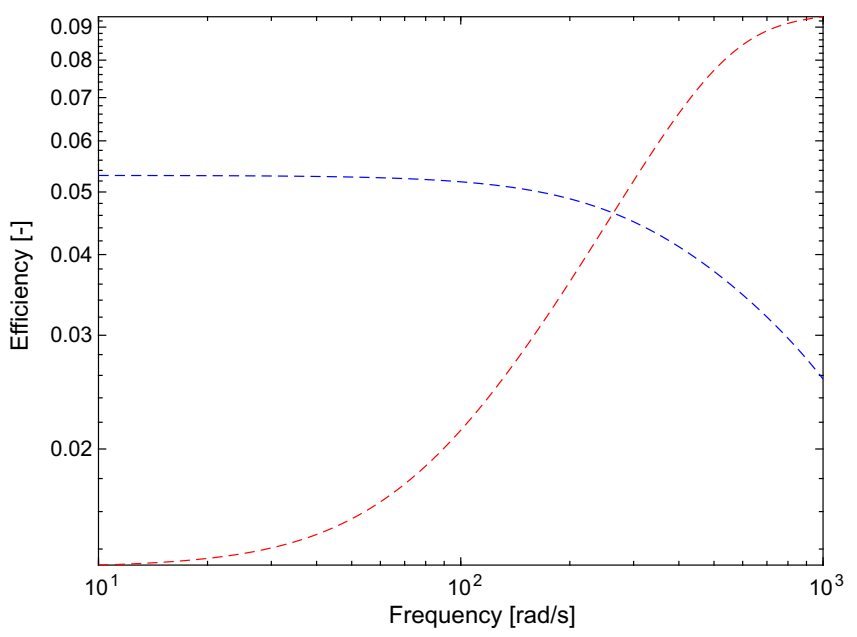

FIGURE 10 Plot of $f_{\alpha}(\omega)$ for $\theta_{1}$ of -0.1 (blue) and for $\theta_{2}$ of -0.1 (red) [Colour figure can be viewed at wileyonlinelibrary.com] 


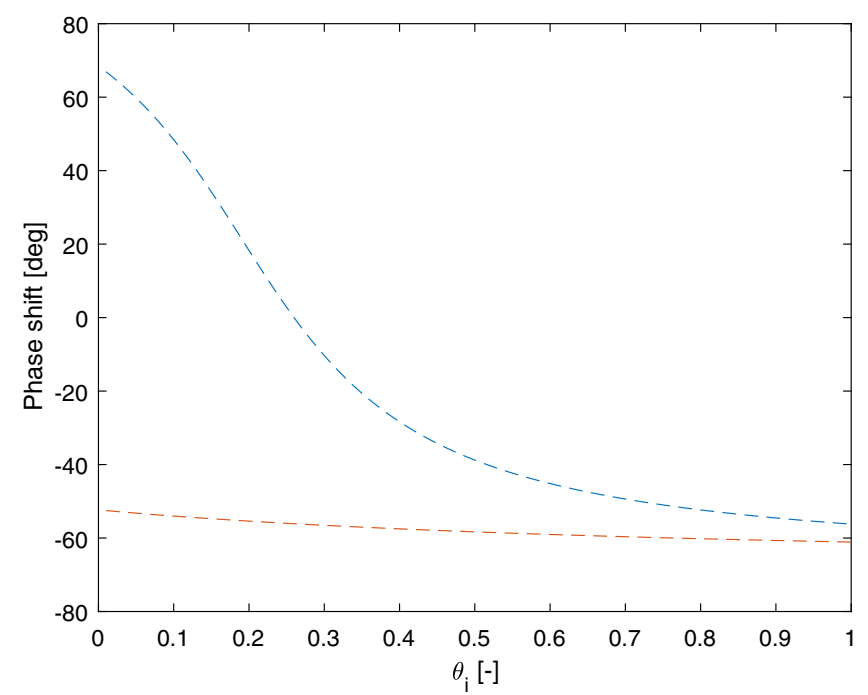

FIGURE 11 The phase shift is shown for each fault from the fault-free case to the case of a complete failure [Colour figure can be viewed at wileyonlinelibrary.com]

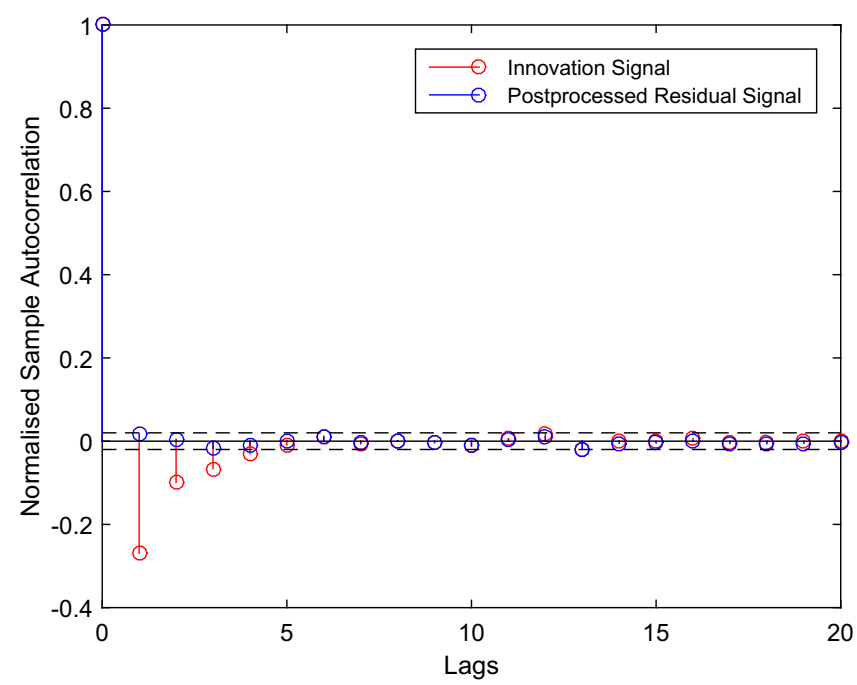

FIGURE 12 Autocorrelation plot of the innovation signal and residual signal, respectively. The circles show the normalised autocorrelation for the respective sample delay. The black stippled lines show the $95 \%$ confidence bounds [Colour figure can be viewed at wileyonlinelibrary.com]

example. Using Equation (30), the energy in the detector signal can be determined, as shown in Equation (61), where $A_{0}$ is found using Equation (23).

$$
\nu=N \frac{A_{0}^{2}}{2}=200 \frac{0.29^{2}}{2}=8.3
$$

With the energy and the variance of the signal determined, the threshold is found using Equation (29) as

$$
\gamma=\sqrt{\sigma^{2} \nu} Q^{-1}\left(P_{F A}\right)=\sqrt{0.14 \cdot 8.3} Q^{-1}(0.0001)=4 .
$$

The fault on either parameter 1 or 2 is expected to be a $10 \%$ reduction in the gain, which result in a 48.4 degree phase shift of the detector signal if it is fault 1 and a 306 degree phase shift if it is fault 2 . The set of possible phase shifts of the detector signal is limited to the set of no fault or a $10 \%$ reduction in either of the parameters. The set of possible phase shifts is thus

$$
\tau=\{0,48.4,306\}
$$


This is a very limited set and could easily be expanded. It is however for the sake of clarity chosen to be rather small in this example.

In case the magnitude of the possible fault is unknown, the set $\tau$ is simply expanded to cover the fault range in an appropriate manner. Given a window length of 200 samples and a sinusoidal excitation signal with an amplitude of 0.1, for a fault $\theta_{1}$ occurring at 5 seconds, the decision algorithm Equation (27) produces decisions, as shown in Figure 13.

With the decision algorithm indicating a fault after 5 seconds, the threshold is determined such that one false alarm is on average happening within the simulation period. The threshold is then calculated using Equation (29), and detection of a fault is shown in Figure 14A.

It is possible to increase the probability of detection simply by increasing the window length. However, it is important to notice that the window length directly imposes a delay between the occurrence of a fault and the detection of it. The window length should therefore be chosen in accordance with the tolerable delay of detection.

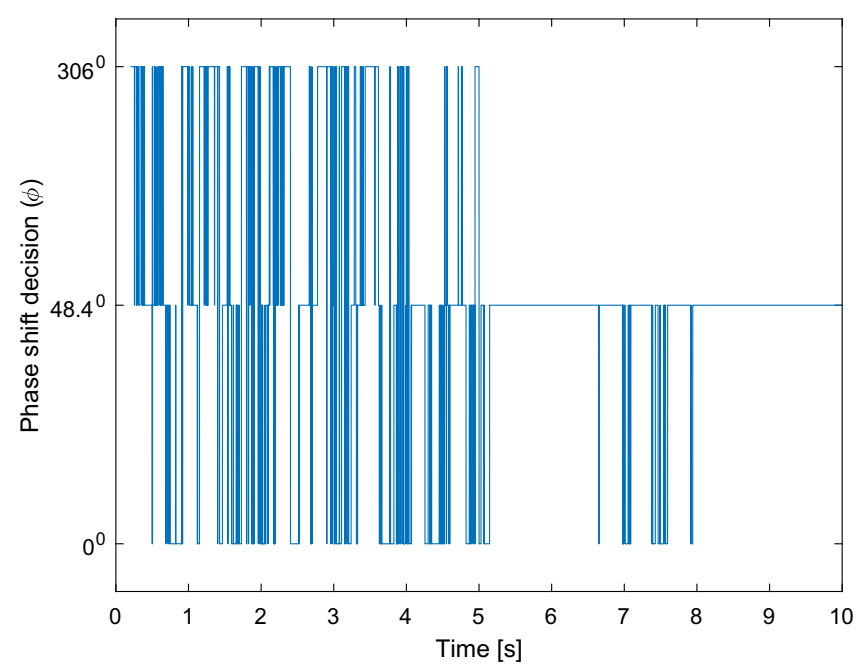

FIGURE 13 Plot of the decision at each time step. The possible phase shifts have been chosen such that a fault of $10 \%$ in either $\theta_{1}$ or $\theta_{2}$ or no fault at all are searched for. A fault is occurring at $5 \mathrm{~s}$ on $\theta_{1}$ [Colour figure can be viewed at wileyonlinelibrary.com]

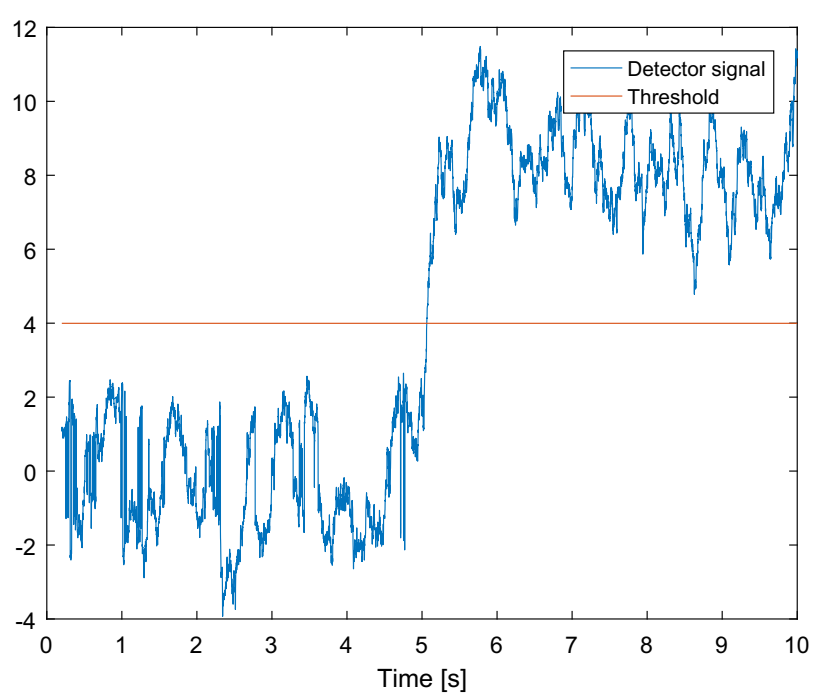

(A)

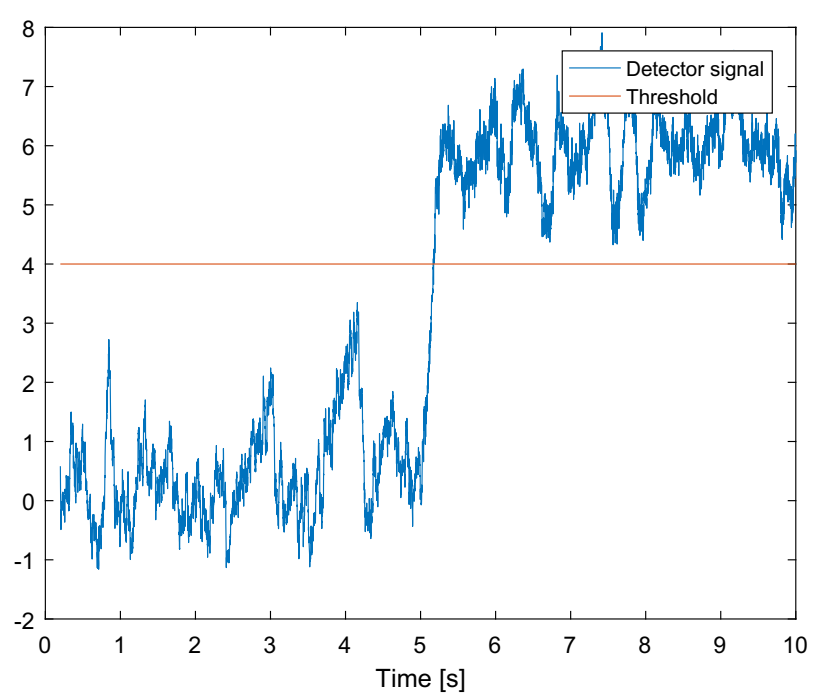

(B)

FIGURE 14 A, Plot of the test statistic given fault $\theta_{1}$ occurring after $5 \mathrm{~s}$; B, Plot of the test statistic given fault $\theta_{2}$ occurring after $5 \mathrm{~s}$ [Colour figure can be viewed at wileyonlinelibrary.com] 


\section{1 | Fault detection with uncertainty}

The example system above is now used to show the impact of parametric uncertainties. The system is modified with a parametric uncertainty of the first parameter in the system matrix. The system is thus redefined by the new system matrix

$$
A=\left[\begin{array}{cc}
a_{11}(1+\mu) & a_{12} \\
a_{21} & a_{22}
\end{array}\right]=\left[\begin{array}{cc}
1+\mu & -0.3 \\
-0.5 & 0.2
\end{array}\right] .
$$

This is the same system as used in the first example with the addition of the small parametric uncertainty $\mu$. The bound of the uncertainty is given as $|\mu|=0.01$. Again, parametric faults of the gains from the input to the 2 states are considered, as shown in Equation (57). The augmented system taking the faults and uncertainty into account is

$$
\begin{aligned}
& {\left[\begin{array}{c}
z \\
\Delta y \\
y
\end{array}\right]=\left[\begin{array}{cc|cccc}
a_{11} & a_{12} & b_{1} & 0 & b_{1} & b_{1} \\
a_{21} & a_{22} & 0 & b_{2} & 0 & b_{2} \\
\hline 0 & 0 & 0 & 0 & 0 & 1 \\
0 & 0 & 0 & 0 & 0 & 1 \\
1 & 0 & 0 & 0 & 0 & 0 \\
c_{1} & c_{2} & 0 & 0 & 0 & 0
\end{array}\right]\left[\begin{array}{c}
w \\
\Delta u \\
u
\end{array}\right]} \\
& {\left[\begin{array}{c}
w \\
\Delta u
\end{array}\right]=\left[\begin{array}{ccc}
\theta_{1} & 0 & 0 \\
0 & \theta_{2} & 0 \\
0 & 0 & \mu
\end{array}\right]\left[\begin{array}{c}
z \\
\Delta y
\end{array}\right] .}
\end{aligned}
$$

Here, Equation (66) presents the faults and uncertainties as a single complete diagonal system with the vectors $z$ and $w$ having an entry for each of the 2 faults considered. This uncertainty introduces an uncertainty region for the phase shift and the amplitude of the residual. However, for a properly identified system, the impact of the uncertainties on the phase shift and amplitude should always be insignificant compared with the impact of the fault in the faulty case. An analysis, however, is possible to determine the phase shift regions of each fault when taking the bounds of the uncertainties into account. In Figure 15, the bounds of the phase shift is shown for faults $\theta_{1}$ and $\theta_{2}$.

From Figure 15, it is clear that as the magnitude of the fault is increased the impact of the uncertainty on the phase shift decreases as expected. In order to determine the threshold such that the probability of a false alarm does not exceed the predetermined value from the previous example the energy added due to the uncertainty is calculated. The bias added to the threshold due to the uncertainty is found, using Equation (67), to be 0.1, which guarantees that the number of false alarms does not exceed the previously determined acceptable limit.

$$
\begin{aligned}
& v_{\mu}=N \frac{\left(A_{0} A_{S_{\mu}}\right)^{2}}{2}=0.1 \\
& \gamma^{\prime}=\gamma+v_{\mu}=4.1
\end{aligned}
$$

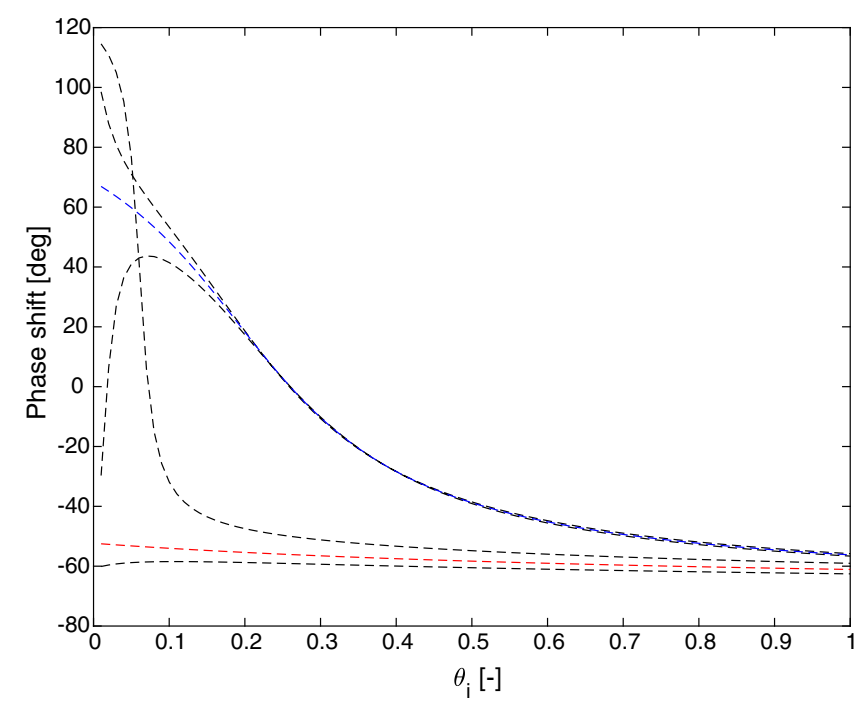

FIGURE 15 Plot of the predicted phase shift of the residual signal $r$ given the fault $\theta_{1}$ (blue) or the fault $\theta_{2}$ (red). The black dashed lines represent the bounds on the phase shift given the uncertainty bound, and the red and blue lines are the phase shift introduced by the fault when the uncertainty is not considered [Colour figure can be viewed at wileyonlinelibrary.com] 


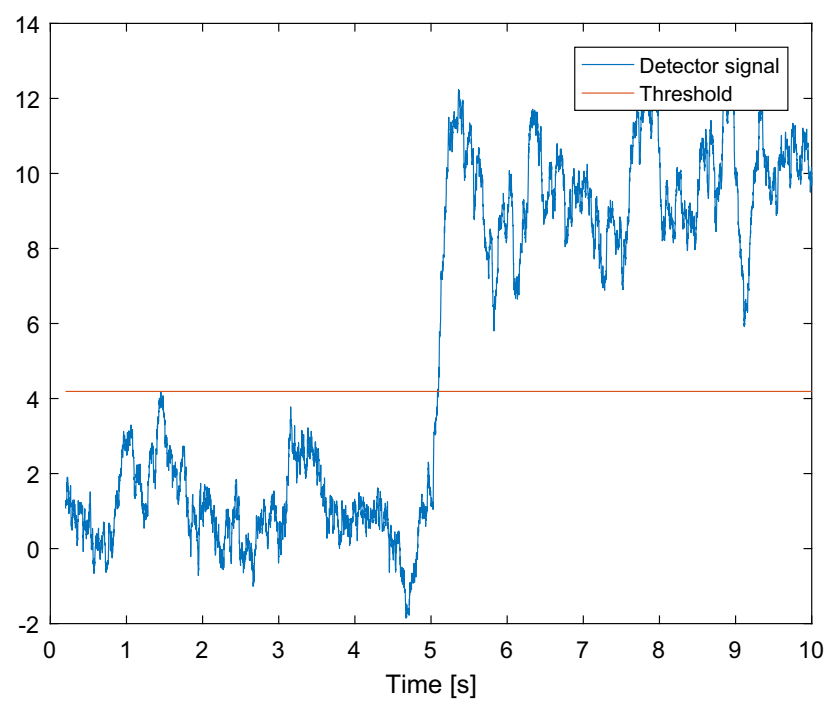

(A)

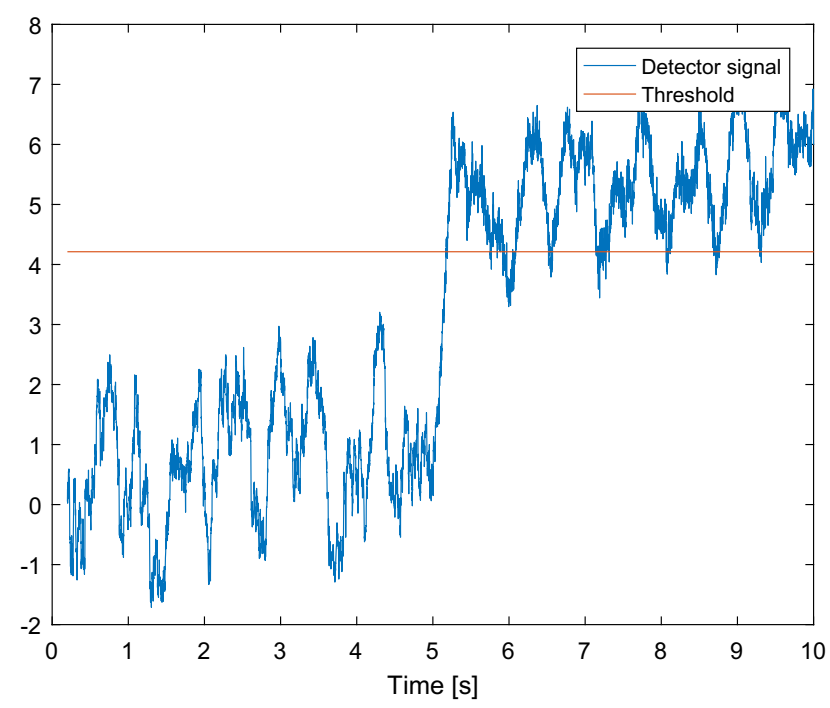

(B)

FIGURE 16 A, Plot of the test statistic given the uncertainty on the system matrix and the fault $\theta_{2}$ occurring after $5 \mathrm{~s}$; B, Plot of the test statistic given the uncertainty on the system matrix and the fault $\theta_{1}$ occurring after $5 \mathrm{~s}$ [Colour figure can be viewed at wileyonlinelibrary.com]

With the reevaluated threshold, the decision response is shown in Figure 16A. Here, the set of possible phase shifts has been kept the same as in the previous example since the 2 possible faults are still clearly separated.

The addition to the threshold using Equation (67) is based on the assumption that the noise is white for the fault-free case, which is not true due to the uncertainty. However, the uncertainty in this example has a relatively insignificant impact on the whiteness of the noise, and thus, the approximation works.

It may be noticed that the threshold is hardly increased due to the uncertainty. This is because the uncertainty introduced in the example has a much larger impact on the phase shift than the magnitude and was thus chosen with a magnitude that provides almost no energy to the detector. It is still possible to distinguish fault from no fault but the variance of the detector signal has increased due to the uncertainty. The example shows that the ability to detect and isolate faults is reduced and a bit more involved when an imperfect model has to be taken into account.

\section{CONCLUSION}

A method for design of an optimal detector for active fault detection of a plant in a closed-loop system was presented in this paper. It was shown possible to decorrelate the noise for both open-loop stable and unstable systems. This made it possible to design optimal detectors based on the Neyman-Pearson detector. Furthermore, it was shown how the detector design makes it possible to isolate parametric faults using only a single excitation signal by exploiting knowledge about the phase shift and amplitude of the fault detection signal. A general method was proposed for detector design, which could cope with multiple possible parametric faults, and a special case was given that greatly simplified the deflection coefficient. Finally, a simulation example of an open-loop unstable system was given. It was shown possible for such a system to generate a residual signal with white noise and design a detector able to both isolate and detect faults of the system for both a system with and without uncertainties.

\section{ORCID}

A. K. Sekunda http://orcid.org/0000-0002-0094-0743
H. H. Niemann (1) http://orcid.org/0000-0002-1518-4979

\section{REFERENCES}

1. Blanke M, Kinnaert M, Lunze J, Staroswiecki M, Schröder J. Diagnosis and Fault-Tolerant Control. Vol. 3. Berlin, Germany: Springer-Verlag; 2016. 
2. Isermann R. Model-based fault-detection and diagnosis-status and applications. Annu Rev Control. 2005;29(1):71-85.

3. Frank P, Ding S, Marcu T. Model-based fault diagnosis in technical processes. Trans Inst Meas Control. 2000;22(1):57-101.

4. Punačocháŕ I, Široky J, Šimandl M. Constrained active fault detection and control. IEEE Trans Autom Control. 2015;60(1):253-258.

5. Kerestecioğlu F, Cetin I. Optimal input design for the detection of changes towards unknown hypotheses. Int J Syst Sci. 2004;35(7):435-444.

6. Campbell SL, Horton KG, Nikoukhah R. Auxiliary signal design for rapid multi-model identification using optimization. Automatica. 2002;38(8):1313-1325.

7. Chiang LH, Russell EL, Braatz RD. Fault Detection and Diagnosis in Industrial Systems. Berlin, Germany: Springer Science \& Business Media; 2000.

8. Poulsen N, Niemann H. Active fault diagnosis based on stochastic tests. Int J Appl Math Comput Sci. 2008;18(4):487-496.

9. Niemann H, Stoustrup J. An architecture for fault tolerant controllers. Int J Control. 2005;78(14):1091-1110.

10. Niemann H, Poulsen NK. Estimation of parametric fault in closed-loop systems. Paper presented at: 2015 American Control Conference (ACC), IEEE; 2015; Chicago, IL.

11. Niemann H, Poulsen NK. Active fault detection in MIMO systems. Paper presented at: 2014 American Control Conference (ACC), IEEE; 2014; Portland, OR.

12. Kay SM. Fundamentals of Statistical Signal Processing: Detection Theory. Vol. 2. Upper Saddle River, NJ: Prentice Hall; 1998.

13. Poulsen NK, Niemann HH. Active fault isolation and estimation. Paper presented at: Information Technology Control Theory Fault and System Diagnosis: Control and Computer Science; 2009; Gdańsk, Poland.

14. Sekunda A, Niemann H, Kjølstad N. Active fault detection based on a statistical test. Paper presented at: 2016 3rd Conference on Control and Fault-Tolerant Systems (SysTol), IEEE; 2016; Barcelona, Spain.

15. Marseglia GR, Raimondo DM. Active fault diagnosis: a multi-parametric approach. Automatica. 2017;79:223-230.

16. Vidyasagar M, Schneider H, Francis BA. Algebraic and topological aspects of feedback stabilization. IEEE Trans Autom Control. 1982;27:880-894.

17. Tay TT, Mareels IMY, Moore JB. High Performance Control. Systems \& Control: Foundations \& Applications. Cambridge, MA: Birkhäuser Boston; 1998.

18. Anderson BD. From Youla-Kucera to identification, adaptive and nonlinear control. Automatica. 1998;34:1485-1506.

19. Sekunda A, Niemann H, Poulsen NK, Santos I. Closed loop identification using a modified Hansen scheme. J Phys Conf Ser. 2015;659(1):012009. http://stacks.iop.org/1742-6596/659/i=1/a=012009

20. Youla D, Jabr H, Bongiorno J. Modern Wiener-Hopf design of optimal controllers-Part II: the multivariable case. IEEE Trans Autom Control. 1976;21(3):319-338.

21. Niemann H. Dual Youla parameterisation. IET Control Theory Appl. 2003;150(5):493-497.

22. Gustafsson F. Adaptive Filtering and Change Detection. Vol. 1. Chichester, UK: John Wiley \& Sons; 2000.

23. Niemann H. A YJBK based architecture for fault diagnosis and fault-tolerant control, linear system theory; 2015.

24. Basseville M, Nikiforov IV. Detection of Abrupt Changes: Theory and Application. Vol. 104. Englewood Cliffs, NJ: Prentice Hall; 1993.

25. Packard A, Doyle J. The complex structured singular value. Automatica. 1993;29(1):71-109.

How to cite this article: Sekunda AK, Niemann HH, Poulsen NK. Detector design for active fault diagnosis in closed-loop systems. Int J Adapt Control Signal Process. 2018;1-18. https://doi.org/10.1002/acs.2858 